\title{
Primer reporte de la colección de moluscos presentes en el Museo Maggiorino Borgatello (Punta Arenas, Chile)
}

\section{First report of the collection of molluscs present in the Maggiorino Borgatello Museum (Punta Arenas, Chile)}

\author{
Sebastián Rosenfeld ${ }^{* 1,2}$, Johanna Marambio ${ }^{1,2} \&$ Cristian Aldea ${ }^{3,4}$ \\ ${ }^{1}$ Laboratorio de Macroalgas Antárticas y Subantárticas, Universidad de Magallanes, Punta Arenas. \\ ${ }^{2}$ Instituto de Ecología y Biodiversidad (IEB), Santiago. \\ ${ }^{3}$ Programa GAIA-Antártica, Universidad de Magallanes. \\ ${ }^{4}$ Laboratorio de Ecología y Medio Ambiente, Instituto de la Patagonia, Universidad de Magallanes. \\ *Autor corresponsal, e-mail:rosenfeld.sebastian@yahoo.com
}

\begin{abstract}
RESUMEN
La colección de moluscos chilenos del Museo Salesiano Maggiorino Borgatello (Punta Arenas, Chile) fue creada alrededor del año 1905 con el objetivo de rescatar el conocimiento local de la malacofauna. Desde esa fecha hasta mediados del siglo XX se habían ingresado numerosos especimenes, los cuales fueron identificados por curadores locales, pero que nunca fueron sometidos a un inventario exhaustivo ni identificación taxonómica. Este trabajo entrega información sobre las especies de moluscos que actualmente están presentes en el museo. Se registraron un total 37 especies de moluscos (26 gasterópodos y 11 bivalvos), de las cuales 19 corresponden a especies de interés comercial. Desde el punto de vista biogeográfico, la colección representa alrededor del $4 \%$ de la malacofauna registrada para el territorio chileno y alrededor del 10\% de la registrada para la Región Magallánica. A nivel de especies, se destaca la presencia de ejemplares de Photinula roseolineata, Calliostoma irisans y Buccinanops paytensis, los cuales presentan muy pocos registros en la provincia Biogeográfica Magallánica. El mantenimiento y conservación de esta colección de moluscos reviste importancia a nivel cultural y científico, como una forma de preservación del patrimonio de esta zona austral del mundo.
\end{abstract}

Palabras clave: Región de Magallanes, Moluscos, Subantártica, Museo

\begin{abstract}
The Chilean mollusc collection of the Maggiorino Borgatello Salesian Museum (Punta Arenas, Chile) was created around the year 1905 aiming to preserve mollusc local knowledge. Since then until mid-twentieth century many specimens had been incorporated into the collection, which were identified by local curators, but never underwent a comprehensive inventory and taxonomic identification. This report provides comprehensive information on the taxonomy and distribution of mollusc species currently present in the museum. We recorded 37 species of molluscs (26 gastropods and 11 bivalves), of which 19 are commercially important species. From the biogeographical standpoint, the collection represents about 4\% of the registered molluscs for the Chilean territory and about $10 \%$ of that reported for the Magellan Region. At the species level, we highlight the presence of specimens of Photinula roseolineata, Calliostoma irisans and Buccinanops paytensis, which have very few records in the Magellanic Biogeographic Province. The maintenance and preservation of this mollusc collection has great local and regional relevance as a way of preserving cultural heritage of this southern part of the world.
\end{abstract}

KeYwords: Magellan Region, Mollusca, sub Antarctic, Museum

\section{INTRODUCCIÓN}

Durante los dos últimos siglos, las colecciones científicas se consideran componentes esenciales de la investigación, en particular para taxónomos y sistemáticos (Suarez \& Tsutsui 2004). Aún nos encontramos en esta etapa de descubrimiento, y la mayoría de las especies que existen en nuestro planeta y sus funciones no han sido descritas (Wilson 2003). Por esta razón, la preservación de las colecciones toma importancia como testimonio de la biodiversidad del planeta, las cuales son fundamentales en la generación y validación del conocimiento científico. 
Dentro de los hitos importantes en el territorio de Magallanes y Tierra del Fuego, fue el establecimiento de los sacerdotes salesianos entre los años 1887 y 1892. Este suceso marcó un antes y después en la historia de la región. La fundación iglesias y las primeras misiones tanto en Chile como en Argentina, generó que su principal objetivo fuese el rescate de la etnografía de las etnias magallánicas. Debido a esto, empiezan a surgir colecciones etnográficas naturales, de fósiles y minerales, las cuales posteriormente fueron intercambiadas con otros museos con el fin de promocionar científicamente a la región de Magallanes. Concomitantemente, el 18 de Septiembre de 1893 se concreta el proyecto "Museo Territorial" el cual sería el encargado de recopilar todas las colecciones y la información existente hasta el momento de la región. Este proyecto fue fundado y dirigido por R. P. Maggiorino Borgatello, siendo él uno de los principales gestores de la colección étnica. Ese mismo año llega a la Misión de Magallanes el coadjutor salesiano y naturalista autodidacta, Don Angel Gaudencio Benove Brizio, el cual comienza con la clasificación y formación de nuevas colecciones naturales. Posteriormente la dirección del museo fue llevada por el Padre Vicente Lucchelli Rolandi, quien fue el encargado de la conservación y registro de las colecciones hasta el día de su muerte (22 de febrero de 1999). Entre las colecciones naturales existentes, se encuentran principalmente la de mamíferos, aves, plantas e invertebrados. Dentro de estas últimas destacan la colección de moluscos y artrópodos. No obstante, queremos destacar que en particular la colección de moluscos constituye a la única formalmente conocida en estas latitudes, siendo formada por los sacerdotes salesianos y coadjuntores de la época, utilizando los protocolos museológicos de esos tiempos, pero que lamentablemente estuvo guardada mucho tiempo, ocasionando pérdidas de ejemplares y la no actualización de la sistemática de las especies. Finalmente, también es importante mencionar que en general los moluscos han sido un grupo muy bien documentado en diversos museos del mundo (e.g. Castellanos 1956, Castellanos \& Fernández 1976, Dijkstra 1999, Schwabe 2010), contribuyendo, de esta manera, a un mejor conocimiento de la malacofauna en distintas regiones del planeta, con el apoyo de estas colecciones (e.g. Zelaya 2004, 2005).

Por consiguiente, el objetivo de este trabajo es entregar el primer reporte de la colección de moluscos presente en el Museo Salesiano Maggiorino Borgatello (Punta Arenas, Chile).

\section{MATERIALES Y MÉTODOS}

Algunos de los ejemplares depositados en el museo fueron reidentificados y otros fueron actualizados sistemáticamente.
Para la identificación taxonómica de las especies, se utilizó la literatura más actualizada de reportes malacológicos en la Provincia Biogeográfica Magallánica (e.g. Reid \& Osorio 2000, Linse 2002), cotejando también con información primordial de trabajos clásicos (e.g. Rochebrune \& Mabille 1889, Smith 1905, Strebel 1905a,b), trabajos sistemáticos sobre taxones específicos (e.g. Allmon 1990, Pastorino, 2005a,b) y bases de información académicas disponibles en Internet (Morris \& Rosenberg 2005, USNM 2010).

Para cada especie identificada se entrega su posición sistemática, las medidas del ejemplar fotografiado y el lugar de colecta de cada ejemplar. Las dimensiones de los gasterópodos se refieren a la altura máxima desde el umbo al borde ventral de la concha y el ancho máximo, perpendicular a la altura; mientras que las medidas de los bivalvos se refieren a la altura máxima desde el umbo al borde ventral de la concha y el ancho máximo entre los márgenes anterior y posterior. Para cada especie se presenta un párrafo de observaciones, considerando aspectos relevantes sobre su taxonomía, morfología comparada con especies similares o cualquier otro aspecto importante de la especie. Todos los ejemplares figurados en este trabajo fueron registrados mediante fotografía digital, utilizando una cámara CANON PowerShot SX40HS y, para los ejemplares más pequeños, una cámara Olympus CAMEDIA C-3030 conectada a un esteroscopio Olympus modelo SZ61.

En esta revisión se presenta primero su distribución en las costas chilenas del Pacifico-Sur y luego su distribución en las costas del Atlántico-Sur.

\section{RESULTADOS}

De un total de 585 ejemplares de moluscos depositados en la colección, se reclasificaron 37 especies, correspondientes a 7 órdenes, 19 familias y 32 géneros. En términos de riqueza por clases, Gastropoda estuvo representada por 26 especies y Bivalvia por 11 especies (Tabla 1).

\section{SistemÁtica \\ Clase Gastropoda \\ Orden Patellogastropoda \\ Familia Nacellidae \\ Género Nacella \\ Nacella mytilina (Helbling 1779)}

(Fig. 1 a-b).

Sinonimia: ver Valdovinos y Rüth (2005).

Lugar de colecta de los ejemplares: Estrecho de Magallanes (Fig. 6)

Distribución: Desde las islas Guarello hasta el cabo de Hornos (Valdovinos \& Rüth 2005). En el océano Atlántico 
desde los $39^{\circ} \mathrm{S}$ (Morris \& Rosenberg 2005) hasta la Isla de los Estados (USNM 2010).

Comentarios: Valdovinos \& Rüth (2005), realizaron una descripción morfológica de las especies del género Nacella en la cual comentaron que Nacella mytilina es claramente diferente al resto de las especies del género. Posteriormente, estudios moleculares realizados para el género (Aranzamendi et al. 2009, González-Wevar et al. 2010, González-Wevar et al. 2011), respaldan la categoría de Nacella mytilina como especie. Es un componente común de la comunidad epibiotica asociada a los bosques de Macrocystis pyrifera de la región de Magallanes (Reid \& Osorio 2000).

\section{Nacella magellanica (Gmelin 1791)}

(Fig. 1c-d).

Sinonimia: ver Valdovinos y Rüth (2005)

Lugar de colecta de los ejemplares: Punta Arenas y Bahía Santa María (Fig. 6)

Distribución: Desde Chiloé hasta el Cabo de Hornos (Valdovinos \& Ruth 2005). En el océano Atlántico desde Bueno Aires (Castellano \& Landoni 1988) hasta la Isla de los Estados (USNM 2010).

Comentarios: Esta especie se caracteriza por presentar una gran variabilidad morfológica de la concha y cuando ciertos ejemplares presentan el ápice inclinado hacia el margen anterior de la concha, pueden estos confundirse con la especie Nacella deaurata (Aranzamendi et al. 2009). Un aspecto histórico importante de Nacella magellanica, es que ha sido reportada como uno de los principales alimentos de algunos grupos de indígenas fueguinos (Osorio et al. 1979).

\section{Nacella deaurata (Gmelin 1791)}

(Fig. 1 e-f).

Sinonimia: ver Valdovinos y Rüth (2005).

Lugar de colecta de los ejemplares: Punta Arenas (Fig. 6)

Distribución: Desde Chiloé hasta las Islas Diego Ramirez (Valdovinos \& Rüth 2005). En el océano Atlántico desde los $38^{\circ} \mathrm{S}$ hasta los $56^{\circ} \mathrm{S}$ (Morris \& Rosenberg 2005).

Comentarios: Según la clasificación realizada por Valdovinos \& Rüth (2005), la morfología de la concha de Nacella deaurata presentaba una gran similitud con la especie Nacella delicatissima posteriormente, Aranzamendi et al. (2009), basándose en técnicas moleculares, concluyeron que los especimenes de $N$. delicatissima se presentaron combinados con los especimenes de $N$. magellanica y $N$. deaurata, sugiriendo que $N$. delicatissima es más bien un morfotipo infrecuente de cada una de esas dos especies.

\section{Nacella flammea (Gmelin 1791)}

(Fig. 1 g-h).

Sinonimia: ver Valdovinos \& Rüth (2005).

Lugar de colecta de los ejemplares: Punta Arenas (Fig. 6)

Distribución: Desde Aysén hasta el Estrecho de Magallanes (Valdovinos \& Rüth 2005).
Comentarios: Esta especie, al igual que Nacella mytilina, habita ambientes submareales y presenta una morfología de la concha distinta al resto de las especies del género (Valdovinos \& Rüth 2005). Habita principalmente sobre fondos rocosos, ramonenado microalgas, diatomeas y film bacteriano (González-Wevar et al. 2011).

Orden Vetigastropoda

Familia Fissurellidae

\section{Género Fissurella}

Fissurella picta (Gmelin 1791)

(Fig. 1 i).

Sinonimia: ver McLean (1984).

Lugar de colecta de los ejemplares: Tierra del Fuego (Fig. 6) Distribución: Desde Valparaíso (Húpe 1984) hasta Bahía Orange (Rochebrune \& Mabille, 1889). En el océano Atlántico Islas Malvinas/Falkand (Melvill \& Standen 1914). Comentario: Aldea \& Rosenfeld (2011), comentaron que en el Pacifico Sur-Oriental, se pueden reconocer dos subespecies geográficas: F. picta picta (Gmelin 1791) y F. picta lata Sowerby, 1835, esta última de distribución más septentrional (McLean 1984). La especie habita principalmente en rocas y grietas que se encuentran desde el eulitoral medio al bajo (Reid \& Osorio 2000).

\section{Fissurella oriens Sowerby 1835}

(Fig. 1 j).

Sinonimia: ver McLean (1984).

Lugar de colecta de los ejemplares: Islas Malvinas/Falkland (Fig. 6)

Distribución: Desde Valparaíso (Húpe 1854) hasta Cabo de Hornos (Rochebrune \& Mabille 1889). En el Atlántico sur: Islas Malvinas/Falkland (Powell 1951).

Comentario: Esta especie se puede distinguir de Fissurella picta, por la ausencia de costillas radiales y por ocupar un hábitat más protegido (Reid \& Osorio 2000). Es común encontrarla en pozas de marea y fondos rocosos, hasta una profundidad de $30 \mathrm{~m}$ (McLean 1984).

\section{Género Fissurellidea}

Fissurellidea patagonica (Strebel 1907)

(Fig. 1 k).

Sinonimia: ver Dell (1971).

Lugar de colecta de los ejemplares: Estrecho de Magallanes (Fig. 6)

Distribución: Desde los $41-56^{\circ} \mathrm{S}$. (Zelaya 2009). En el océano Atlántico desde los $35^{\circ} \mathrm{S}$ hasta los $55^{\circ} \mathrm{S}$ (Morris \& Rosenberg 2005).

Comentario: Esta especie, a diferencia de las especies de Fissurella, presenta un foramen más grande, llegando a ocupar desde 1/4 a 1/3 del largo de la concha (Zelaya 2009). Es común encontrarla en lugares rocosos; desde el intermareal inferior hasta los $70 \mathrm{~m}$ de profundidad (Zelaya 2009). 
Familia Tegulidae

\section{Género Tegula}

Tegula atra (Lesson 1831)

(Fig. $1 \mathrm{~m}$ ).

Sinonimia: ver Reid \& Osorio (2000).

Lugar de colecta de los ejemplares: Punta Arenas (Fig. 6)

Distribución: Desde los $7^{\circ} \mathrm{S}$ hasta los $54^{\circ} \mathrm{S}$. (Reid \& Osorio 2000). En el océano Atlántico desde los $38^{\circ} \mathrm{S}$ hasta los $55^{\circ} \mathrm{S}$ (Morris \& Rosenberg 2005).
Comentario: Reid \& Osorio (2000), describen este gasterópodo por su característico color negro mate - violeta con el interior blanco nacarado. Los mismos autores mencionaron que esta especie es muy es abundante en las costas rocosas, cuya exposición va desde protegida a moderada; encontrándose principalmente sobre y debajo de piedras y en los acantilados. Es común encontrarla en zonas de Lithothamnion sp. y en los bosques de Lessonia sp.

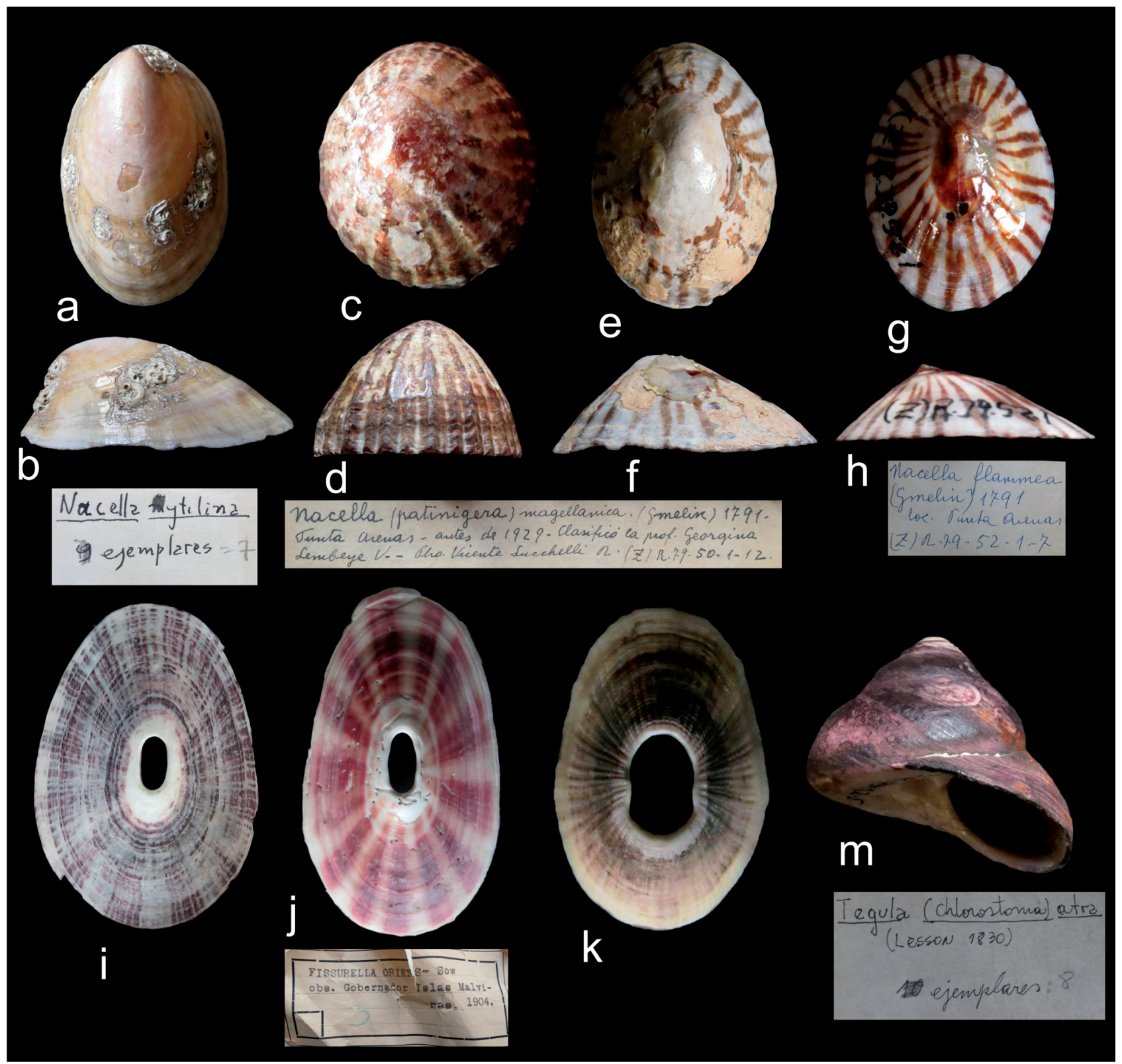

Figura 1. a-b) Nacella mytilina (55 mm; $35 \mathrm{~mm} ; 25 \mathrm{~mm})$, c-d) Nacella magellanica (48 mm; $37 \mathrm{~mm} ; 32 \mathrm{~mm})$, e-f) Nacella deaurata (38 $\mathrm{mm} ; 27 \mathrm{~mm} ; 15 \mathrm{~mm})$, g-h) Nacella flammea $(23 \mathrm{~mm} ; 19 \mathrm{~mm} ; 9 \mathrm{~mm})$ ( i) Fissurella picta $(73 \mathrm{~mm} ; 55 \mathrm{~mm} ; 25 \mathrm{~mm})$, j) Fissurella oriens $(60$ mm; $30 \mathrm{~mm} ; 14 \mathrm{~mm})$, k) Fissurellidea patagonica $(24 \mathrm{~mm} ; 15 \mathrm{~mm} ; 4 \mathrm{~mm}), \mathrm{m})$ Tegula atra (40 mm; $55 \mathrm{~mm})$.

Figure 1. a-b) Nacella mytilina (55 mm; $35 \mathrm{~mm} ; 25 \mathrm{~mm}$ ), c-d) Nacella magellanica (48 mm; $37 \mathrm{~mm} ; 32 \mathrm{~mm})$, g-h) Nacella deaurata (38 mm; $27 \mathrm{~mm} ; 15 \mathrm{~mm})$, i) Fissurella picta $(73 \mathrm{~mm} ; 55 \mathrm{~mm} ; 25 \mathrm{~mm})$, j) Fissurella oriens (60 mm; $30 \mathrm{~mm} ; 14 \mathrm{~mm})$, k) Fissurellidea patagonica $(24 \mathrm{~mm} ; 15 \mathrm{~mm} ; 4 \mathrm{~mm}), \mathrm{m})$ Tegula atra $(40 \mathrm{~mm} ; 55 \mathrm{~mm})$. 
TABLA. 1. Lista sistemática de todas las especies de moluscos marinos depositadas en el Museo Maggiorino Borgatello, Punta Arenas, Chile. Table 1. Systematic list of all species of marine mollusks deposited in the Museo Maggiorino Borgatello, Punta Arenas, Chile.

\begin{tabular}{|c|c|}
\hline TAXÓN & ESPECIE \\
\hline \multicolumn{2}{|l|}{ GASTROPODA } \\
\hline \multicolumn{2}{|c|}{ Orden $\sim$ Patellogastropoda } \\
\hline \multirow[t]{4}{*}{ Nacellidae } & Nacella deaurata (Gmelin, 1791) \\
\hline & Nacella flammea (Gmelin, 1791) \\
\hline & Nacella mytilina (Helbling, 1779) \\
\hline & Nacella magellanica (Gmelin, 1791) \\
\hline \multicolumn{2}{|c|}{ Orden $\sim$ Vetigastropoda } \\
\hline \multirow[t]{3}{*}{ Fissurellidae } & Fissurella picta (Gmelin, 1791) \\
\hline & Fissurella oriens Sowerby, 1835 \\
\hline & Fissurellidea patagonica (Strebel, 1907) \\
\hline Tegulidae & Tegula atra (Lesson, 1831) \\
\hline \multirow[t]{5}{*}{ Calliostomatidae } & Margarella violacea (King y Broderip, 1832) \\
\hline & Margarella expansa ( Sowerby, 1838) \\
\hline & Calliostoma irisans Strebel, 1905 \\
\hline & Photinula roseolineata Smith, 1905 \\
\hline & Photinastoma taeniatum (Sowerby, 1825) \\
\hline \multicolumn{2}{|c|}{ Orden $\sim$ Littorinimorpha } \\
\hline \multirow[t]{2}{*}{ Calyptraeidae } & Crepipatella cf. Dilatata Lamarck, 1822 \\
\hline & Trochita pileus (Lamarck, 1822) \\
\hline Naticidae & Falsilunatia soluta (Gould, 1848) \\
\hline Ranellidae & Fusitriton magellanicus Röding, 1798) \\
\hline \multicolumn{2}{|c|}{ Orden $\sim$ Neogastropoda } \\
\hline Buccinidae & Pareuthria plumbea (Philippi, 1844) \\
\hline Nassariidae & Buccinanops paytensis (Kiener, 1834) \\
\hline \multirow[t]{4}{*}{ Muricidae } & Xymenopsis muriciformis ( King y Broderip, 1832) \\
\hline & Trophon geversianus (Pallas, 1774) \\
\hline & Concholepas concholepas (Bruguiere, 1789) \\
\hline & Acanthina monodon (Pallas, 1774) \\
\hline \multirow[t]{2}{*}{ Volutidae } & Odontocymbiola magellanica (Gmelin, 1791) \\
\hline & Adelomelon ancilla (Lightfoot, 1786) \\
\hline \multicolumn{2}{|c|}{ Orden $\sim$ Cladobranchia } \\
\hline Siphonariidae & Siphonaria lesonii Blainville, 1827 \\
\hline \multicolumn{2}{|l|}{ BIVALVIA } \\
\hline \multicolumn{2}{|l|}{ Orden $\sim$ Pteriomorphia } \\
\hline \multirow[t]{3}{*}{ Mytilidae } & Mytilus edulis platensis d’ Orbigny, 1842 \\
\hline & Choromytilus chorus (Molina, 1782) \\
\hline & Aulacomya atra (Molina, 1782) \\
\hline \multirow[t]{2}{*}{ Pectinidae } & Zygochlamys patagonica (King y Broderip, 1832) \\
\hline & Aequipecten tehuelchus (d' Orbigny, 1842) \\
\hline \multicolumn{2}{|l|}{ Orden $\sim$ Heterodonta } \\
\hline Mactridae & Mulinia edulis (King \& Broderip, 1832) \\
\hline Pharidae & Ensis macha (Molina, 1782) \\
\hline \multirow[t]{3}{*}{ Veneridae } & Ameghinomya antiqua (King \& Broderip, 1832) \\
\hline & Tawera elliptica (Hupé en gay, 1854) \\
\hline & Retrotapes exalbidus (Dillwyn, 1817) \\
\hline
\end{tabular}


Familia Calliostomatidae

\section{Género Margarella}

Margarella violacea (King \& Broderip, 1832)

(Fig. 2 a).

Sinonimia: ver (Dell 1971).

Lugar de colecta de los ejemplares: Estrecho de Magallanes (Fig. 6)

Distribución: Desde los $46^{\circ} \mathrm{S}$ (Reid \& Osorio 2000) hasta los $56^{\circ} \mathrm{S}$ (Linse 2002). En el Atlántico en las Islas Malvinas/ Falkland (Powell 1951) hasta la Isla de los Estados (USNM, 2010).

Comentario: Esta especie es un componente común de los fondos sublitorales del Estrecho de Magallanes, habiéndose reportado como el molusco más abundante en trabajos bentónicos del Estrecho de Magallanes (ver Ríos et al. 2003, Aldea et al. 2011, Rosenfeld et al. 2015). King \& Broderip (1832) comentaron que los indígenas de la zona utilizaban las conchas para fabricarse collares.

\section{Margarella expansa (Sowerby, 1838)}

(Fig. 2 b).

Sinonimia: ver Powell (1951).

Lugar de colecta de los ejemplares: Canal Beagle (Fig. 6) Distribución: Estrecho de Magallanes en Buque Quemado (Aldea \& Rosenfeld 2011) hasta Bahía Orange (Lamy 1905). En el océano Atlántico en el Banco Burdwood (Melvill \& Standen 1907).

Comentario: Respecto a su morfología, Rosenfeld et al. (2011) comentaron que las dos capas definidas de la concha, la coloración negra del cuerpo y la presencia de un tentáculo epipodial impar izquierdo adicional son características particulares de la especie. Las demás características morfológicas que presenta la especie, son inherentes de las especies del género estudiadas por Zelaya (2004) en el Suroeste Atlántico y aguas subantárticas y antárticas.

\section{Género Calliostoma}

\section{Calliostoma irisans Strebel 1905}

(Fig. 2 c).

Sinonimia: ver Castellanos \& Fernández (1976).

Lugar de colecta de los ejemplares: Islas Malvinas/Falkland (Fig. 6)

Distribución: Desde los $50^{\circ} \mathrm{S}$ hasta los $55^{\circ} \mathrm{S}$ (Valdovinos 1999); En el océano Atlántico desde los $45^{\circ} \mathrm{S}$ hasta los $58^{\circ} \mathrm{S}$ (Morris \& Rosenberg 2005).

Comentario: Respecto a su morfología, Castellanos \& Fernández (1976), comentaron que esta especie es similar a Calliostoma nudum (Philippi 1845), pero que difiere de esta última al presentar una espira más alta y nódulos más juntos en la primera vuelta de la teleoconcha. Debido a los bajos reportes de esta especie en Magallanes (Strebel 1905a, Castellanos \& Fernández 1976, Castellanos \& Landoni 1989, Linse 2002), algunos aspectos de su biología y ecología aún siguen siendo desconocidos.

\section{Género Photinula}

Photinula roseolineata Smith 1905

(Fig. 2 d).

Sinonimia: ver Morris \& Rosenberg (2005).

Lugar de colecta de los ejemplares: Islas Malvinas/Falkland (Fig. 6)

Distribución: Tierra del Fuego y Estrecho de Magallanes (Morris \& Rosenberg 2005).

Comentario: En la descripción de esta especie, Smith (1905) comentó que se caracteriza por su color y forma, destacando también la presencia de cuatro líneas espirales de coloración rosada en la penúltima vuelta y 9 líneas espirales en la última vuelta. Es importante mencionar que Photinula roseolineata, no es una especie muy conocida dentro de la región magallánica, debido principalmente a sus bajos registros, siendo registrada únicamente por (Ríos et al. 2003) para la entrada oriental del Estrecho de Magallanes. Al respecto, esta especie no está considerada en el listado de especies de moluscos de la región de Magallanes propuesto por (Linse 1999).

\section{Género Photinastoma}

Photinastoma taeniatum (Sowerby 1825)

(Fig. 2 e).

Sinonimia: ver Powell (1951).

Lugar de colecta de los ejemplares: Islas Malvinas/Falkland y Estrecho de Magallanes (Fig. 6)

Distribución: Estrecho de Magallanes (Ríos et al. 2003). En el océano Atlántico en el Río Santa Cruz (Powell 1951) y en las Islas Malvinas/Falkland (Strebel 1908).

Comentario: Powell (1951), menciona que esta especie presenta líneas espirales rojas sobre una superficie lisa blanca. Este mismo autor, comentó que, tanto esta especie como la subespecie Photinastoma taeniatum nivea, presentan tres cordones espirales en la primera vuelta de la protoconcha.

Orden Littorinimorpha

Familia Calyptraeidae

Género Crepipatella

Crepipatella cf. dilatata Lamarck 1822

(Fig. 2 f).

Sinonimia: ver Morris \& Rosenberg (2005).

Lugar de colecta de los ejemplares: Estrecho de Magallanes (Fig. 6)

Distribución: Desde los $18^{\circ} \mathrm{S}$ hasta los $56^{\circ} \mathrm{S}$ (Zelaya 2009). En el océano Atlántico desde los $35^{\circ} \mathrm{S}$ hasta los $55^{\circ} \mathrm{S}$ (Morris \& Rosenberg 2005).

Comentarios: Los especimenes del museo originalmente estaban clasificados como Crepidula dilatata; no obstante fueron mencionados como especimenes conferidos (cf.), ya que solamente se cuenta con conchas. En este sentido, Gallardo (1979) estableció que las especies $C$. dilatata y $C$. fecunda no se pueden clasificar por su concha, 
diferenciándose por su tipo de desarrollo: C. fecunda presenta desarrollo indirecto, mientras que $C$. dilatata presenta desarrollo directo.

\section{Género Trochita}

Trochita pileus (Lamarck 1822)

(Fig. 2 g).

Sinonimia: ver Linse (2002).

Lugar de colecta de los ejemplares: Estrecho de Magallanes (Fig. 6)
Distribución: Desde los $33^{\circ}$ hasta los $55^{\circ} \mathrm{S}$ (Aldea \& Rosenfeld 2011). En el océano Atlántico desde los $37^{\circ} \mathrm{S}$ (Morris \& Rosenberg 2005) hasta la Isla de los Estados (USNM 2010).

Comentario: Esta especie es muy similar morfológicamente a $T$. pileolus. La diferencia externa más conspicua entre ambas especies es que $T$. pileus presenta una protoconcha más lisa, mientras que $T$. pileolus tiene una protoconcha rugosa. Esta especie presenta un amplio rango batimétrico desde los $0 \mathrm{~m}$ a los $484 \mathrm{~m}$ (Aldea \& Rosenfeld 2011).

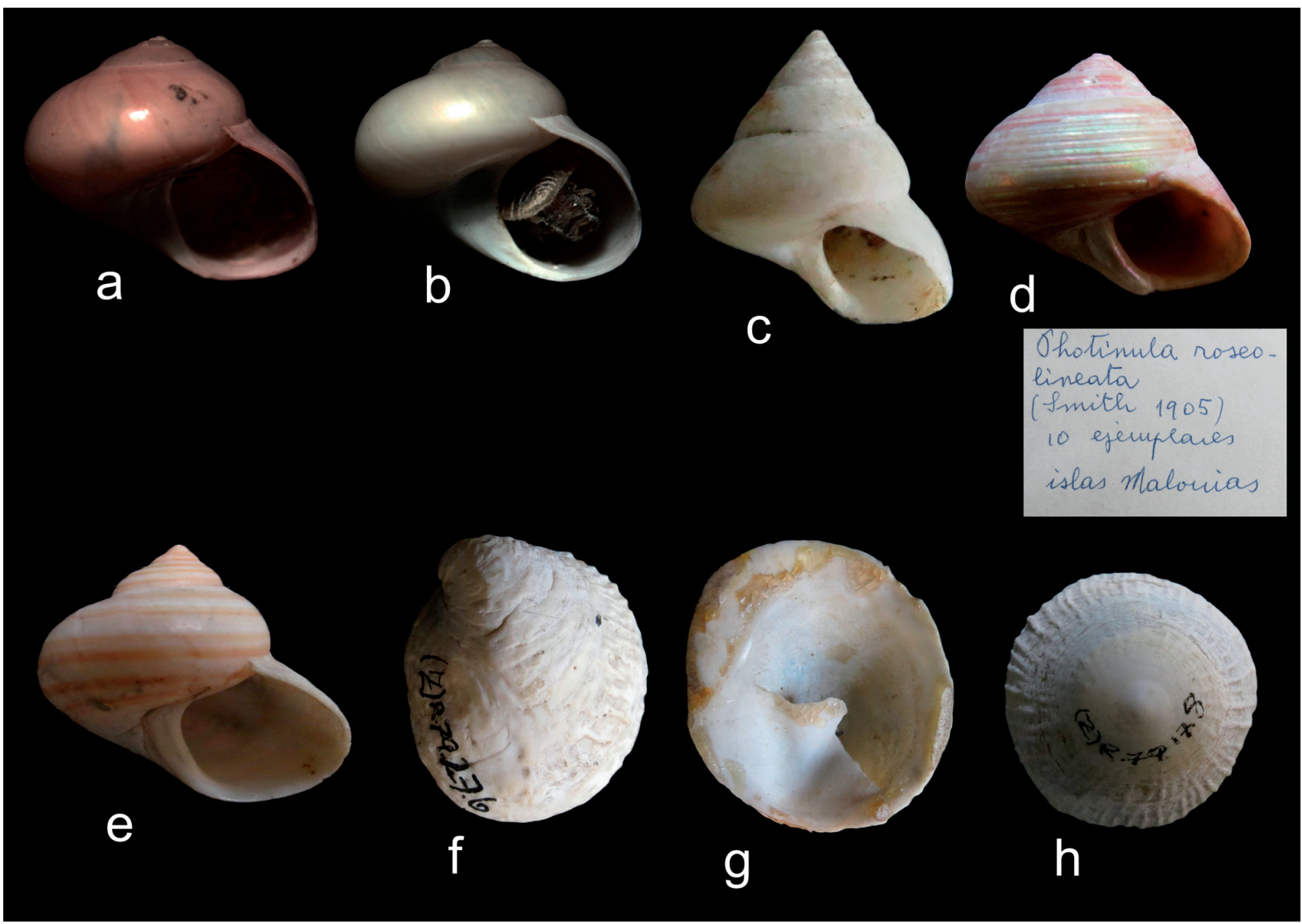

Figura 2. a) Margarella violacea (10 mm; $12 \mathrm{~mm})$, b) Margarella expansa (11 mm; $14 \mathrm{~mm})$, c) Calliostoma irisans (15 mm; $13 \mathrm{~mm})$, d) Photinula roseolineata (14 mm; $16 \mathrm{~mm})$, e) Photinastoma taeniatum (20 mm; $25 \mathrm{~mm})$, f-g) Crepidula dilatata (27 mm; $25 \mathrm{~mm}), \mathrm{h})$ Trochita pileus (28 $\mathrm{mm} ; 13 \mathrm{~mm})$.

Figure 2. a) Margarella violacea (10 mm; $12 \mathrm{~mm})$, b) Margarella expansa (11 mm; $14 \mathrm{~mm})$, c) Calliostoma irisans (15 mm; $13 \mathrm{~mm})$, d) Photinula roseolineata (14 mm; $16 \mathrm{~mm})$, e) Photinastoma taeniatum (20 mm; $25 \mathrm{~mm})$, f-g) Crepidula dilatata (27 mm; $25 \mathrm{~mm})$, h) Trochita pileus (28 mm; $13 \mathrm{~mm})$. 
Familia Naticidae

Género Falsilunatia

Falsilunatia soluta (Gould 1848)

(Fig. 3 a).

Sinonimia: ver Pastorino (2005a).

Lugar de colecta de los ejemplares: Estrecho de Magallanes (Fig. 6)

Distribución: Estrecho de Magallanes (Strebel 1906), Isla Navarino (Strebel 1906; Ojeda et al. 2010). En el océano Atlántico en las Islas Malvinas/Falkland (Pastorino 2005a) hasta la Isla de los Estados (Pastorino 2005a)

Comentario: En la revisión de la familia Naticidae de la costa Patagónica (Pastorino 2005a) se estableció que la morfología de la concha de esta especie, se distingue del resto de las especies de la Naticidae, por presentar una espira mas baja, vueltas convexas con suturas impresas y un callo parietal comprimido.

Familia Ranellidae

Género Fusitriton

\section{Fusitriton magellanicus Röding 1798}

(Fig. 3 b).

Sinonimia: ver Powell (1951).

Lugar de colecta de los ejemplares: Estrecho de Magallanes (Fig. 6)

Distribución: Desde los $32^{\circ} \mathrm{S}$ hasta los $56^{\circ} \mathrm{S}$ (Zelaya 2009). En el océano Atlántico desde los $34^{\circ} \mathrm{S}$ (Morris \& Rosenberg 2005) hasta el Estrecho de Le Maire (Strebel 1905b).

Comentario: Zelaya (2009), comento que morfológicamente esta especie se caracteriza por presentar un periostraco piloso pajizo, amarillento o pardusco. Se le encuentra habitando principalmente en fondos con grava y guijarros, frecuentemente en bancos de mitílidos y también en praderas de Gigartina skottsbergii (Rosenfeld et al. 2015).

Orden Neogastropoda

Familia Buccinidae

Género Pareuthria

Pareuthria plumbea (Philippi 1844)

(Fig. 3 c).

Sinonimia: ver Dell (1971).

Lugar de colecta de los ejemplares: Estrecho de Magallanes (Fig. 6)

Distribución: Desde Puerto Eden hasta el Seno Grandi (Dell 1971). En el oceano Atlántico desde San Sebastián hasta la Isla de los Estados (USNM 2010).

Comentario: En relación a su ciclo de vida, se caracteriza porque, a diferencia de otras especies de la familia Buccinidae, presenta un desarrollo directo, depositando una masa de huevos donde se desarrollan los embriones (Pastorino \& Penchaszadeh 2002). Esta especie habita en substratos rocosos, bajo de rocas, en grietas, paredes rocosas y pozas de marea; tambien sobre algas pardas; desde el intermareal hasta los $80 \mathrm{~m}$ de profundidad (Zelaya 2009).
Familia Nassariidae

Género Buccinanops

Buccinanops paytensis (Kiener 1834)

(Fig. 3 d).

Sinonimia: ver Allmon (1990).

Lugar de colecta de los ejemplares: Estrecho de Magallanes (Fig. 6)

Distribución: En las costas de Perú (Kiener 1834) hasta el estrecho de Magallanes (Allmon 1990). En el océano Atlántico desde los $46^{\circ} \mathrm{S}$ hasta los $54^{\circ} \mathrm{S}$ (Morris \& Rosenberg 2005).

Comentario: A pesar de que esta especie está presente en el Estrecho de Magallanes (Allmon 1990), no ha sido reportada en estudios actuales de moluscos (Mutschke et al. 1998, Linse 2002, Rios et al. 2003, Rios et al. 2007, Aldea \& Rosenfeld 2011, Aldea et al. 2011, Rosenfeld et al. 2015) y no está considerada en el listado de especies de moluscos de la región de Magallanes propuesto por (Linse 1999). Otra especie del género Buccinanops que está presente en el Estrecho de Magallanes es Buccinanops globulosum (Kiener 1834). Sin embargo, Avaca et al. (2010) comentó que esta especie es un componente endémico de la costa del Atlántico Suroeste $\left(34^{\circ} \mathrm{S}-48^{\circ} \mathrm{S}\right)$. No obstante, existe un registro de Allmon (1990), para la zona de Punta Arenas en el Estrecho de Magallanes, por lo tanto, su presencia en el Estrecho de Magallanes aún debe ser estudiada.

Familia Muricidae

Género Xymenopsis

Xymenopsis muriciformis (King \& Broderip 1832)

(Fig. 3 e).

Sinonimia: ver Pastorino y Harasewych (2000).

Lugar de colecta de los ejemplares: Punta Arenas (Fig. 6)

Distribución: Desde Isla de los Chonos (Rochebrune \& Mabille 1889) hasta Cabo de hornos (Strebel 1904). En el oceano Atlántico desde los $41^{\circ} \mathrm{S}$ (Morris \& Rosenberg 2005) hasta la Isla de los Estados (Pastorino \& Harasewych 2000). Comentario: Habita en fondos de grava, guijarros, sobre corales, sobre grampones de Macrocystis pyrifera (Rios et al. 2007). Recientemente, se registró habitando asociada a praderas de Gigartina skottsbergii (Rosenfeld et al. 2015). En su ciclo de vida presenta un desarrollo directo, colocando ovicapsulas en sustratos rocosos (Zelaya 2009).

\section{Género Trophon}

Trophon geversianus (Pallas, 1774)

(Fig. $3 \mathrm{f}$ ).

Sinonimia: ver Pastorino (2005b).

Lugar de colecta de los ejemplares: Estrecho de Magallanes (Fig. 6)

Distribución: Estrecho de Magallanes (Powell 1951) hasta Cabo de Hornos (Rochebrune \& Mabille 1889). En el océano Atlántico desde Buenos Aires (Morris \& Rosenberg 2005) hasta la Isla de los Estados (USNM 2010). 
Comentario: Se ha comentado sobre su variabilidad morfológica (e.g. Pastorino 2005b, Aldea \& Troncoso 2010), encontrándose rangos de formas lisas hasta ornamentadas. Habita en fondos de substratos rocosos, grietas, bancos de mejillones y praderas de Gigartina skottsbergii (Rosenfeld 2012), encontrándose desde el intermareal inferior hasta los $80 \mathrm{~m}$ de profundidad (Zelaya 2009).

\section{Género Concholepas}

Concholepas concholepas (Bruguière 1789)

(Fig. 3 g).

Sinonimia: ver Dell (1971).
Lugar de colecta de los ejemplares: Puerto Edén (Fig. 6) Distribución: Desde los $18^{\circ} \mathrm{S}$ hasta los $55^{\circ} \mathrm{S}$. (Reid \& Osorio 2000).

Comentario: Posee una concha sólida color marrón, con el interior blanco y un margen marrón, con tamaños adultos frecuentares desde unos 100 a 150 mm (Zelaya 2009); ésta presenta una forma esculpida por costillas espirales gruesas. Se le encuentra generalmente en substratos de roca, en la parte superior de la zona de algas calcáreas y en sectores costeros fuertemente expuestos. En Chile central y más al norte, la población adulta es principalmente submareal, pudiendo ser encontradas a profundidades de $40 \mathrm{~m}$ (Reid \& Osorio 2000).

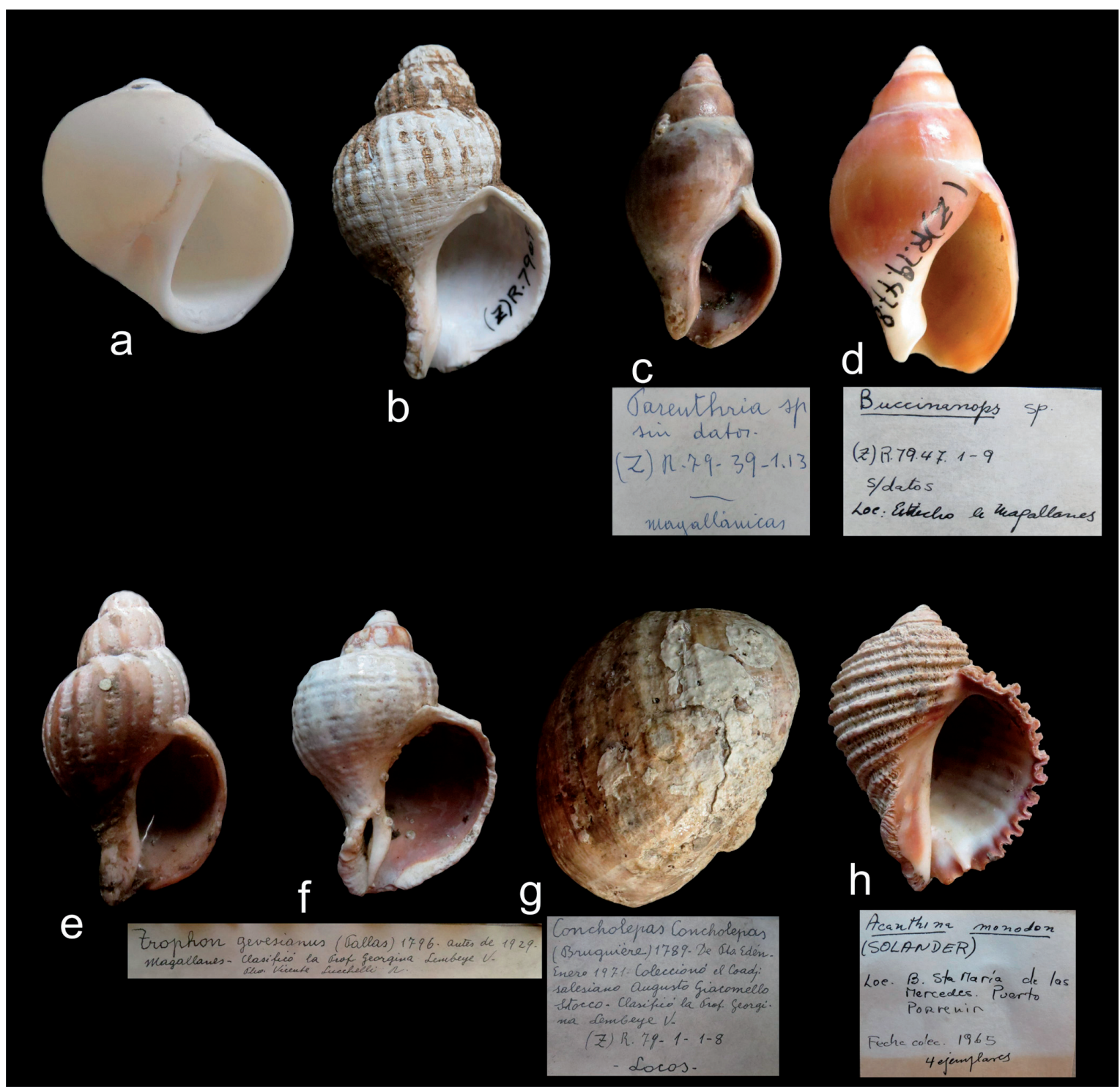

Figura 3. a) Falsilunatia soluta (19 mm; $20 \mathrm{~mm})$, b) Fusitriton magellanicus ( $85 \mathrm{~mm} ; 55 \mathrm{~mm})$, c) Pareuthria plumbea (20 mm; $10 \mathrm{~mm})$, d) Buccinanops paytensis (52 mm; $28 \mathrm{~mm})$, e) Xymenopsis muriciformis $(20 \mathrm{~mm} ; 9 \mathrm{~mm})$, g) Trophon geversianus $(60 \mathrm{~mm} ; 30 \mathrm{~mm})$, h) Acanthina monodon $(40 \mathrm{~mm} ; 30 \mathrm{~mm})$.

Figure 3. a) Falsilunatia soluta (19 mm; $20 \mathrm{~mm})$, b) Fusitriton magellanicus ( $85 \mathrm{~mm} ; 55 \mathrm{~mm})$, c) Pareuthria plumbea (20 mm; $10 \mathrm{~mm})$, d) Buccinanops paytensis (52 mm; $28 \mathrm{~mm})$, e) Xymenopsis muriciformis (20 mm; $9 \mathrm{~mm}), \mathrm{g})$ Trophon geversianus $(60 \mathrm{~mm} ; 30 \mathrm{~mm})$, h) Acanthina monodon $(40 \mathrm{~mm} ; 30 \mathrm{~mm})$. 


\section{Género Acanthina}

Acanthina monodon (Pallas 1774)

(Fig. 3 h).

Sinonimia: ver Dell (1971).

Lugar de colecta de los ejemplares: Bahía Santa María y

Estrecho de Magallanes (Fig. 6)

Distribución: Desde los $22^{\circ} \mathrm{S}$ hasta los $55^{\circ} \mathrm{S}$. (Reid \& Osorio

2000). En el océano Atlántico desde los $51^{\circ} \mathrm{S}$ hasta los $55^{\circ} \mathrm{S}$

(Morris \& Rosenberg 2005).

Comentario: Especie de características polimórficas (Reid \& Osorio 2000), la cual posee un prominente diente en base caracterítico de la especie (Zelaya 2009). Su coloración es variable desde pardo obscura, morado, castaño claro, amarillento o blancuzca.

Familia Volutidae

Género Odontocymbiola

Odontocymbiola magellanica (Gmelin 1791)

(Fig. 4 a).

Sinonimia: ver Morris \& Rosenberg (2005).

Lugar de colecta de los ejemplares: Río Grande (Fig. 6)

Distribución: Desde los $41^{\circ} \mathrm{S}$ hasta los $56^{\circ} \mathrm{S}$ (Zelaya 2009).

En el océano Atlántico desde los $35^{\circ} \mathrm{S}$ hasta los $55^{\circ} \mathrm{S}$ (Morris \& Rosenberg 2005).

Comentario: Esta especie es carnívora, depredando activamente sobre bivalvos y otros gasterópodos; aunque en condiciones particulares también es carroñera (Zelaya 2009). Respecto a su ciclo de vida, los individuos colocan una gran ovicapsula esférica, recubierta externamente por una fina capa calcárea, en su interior se desarrollan 6-12 embriones, que eclosionan como juveniles (Zelaya 2009).

\section{Género Adelomelon}

\section{Adelomelon ancilla (Lightfoot, 1786)}

(Fig. 4 b).

Sinonimia: ver Morris \& Rosenberg (2005).

Lugar de colecta de los ejemplares: Río Grande (Fig. 6)

Distribución: Desde los $42^{\circ} \mathrm{S}$ hasta los $54^{\circ} \mathrm{S}$. (Reid \& Osorio 2000). En el océano Atlántico desde los $29^{\circ} \mathrm{S}$ hasta los $55^{\circ} \mathrm{S}$ (Morris \& Rosenberg 2005).

Comentario: Reid \& Osorio (2000) comentaron que este gasterópodo posee una concha de color crema, en cuyo exterior presenta escasas líneas en formas de zig-zag de color marron obscuro, mientras que el interior es de color naranjo pálido. Zelaya comento que esta especie es muy facil confundirla con el gasterópodo Odontocymbiola magellanica, pero que se pueden diferenciar puesto que este ultima presenta la concha más globosa, la espira más baja y el interior pardusco. Considerando su similitud externa con O. magellanica, Urra et al. (2007) desarrollaron un análisis morfométrico para diferenciar ambas especies. Se le encuentra entre los 10 a los $600 \mathrm{~m}$ de profundidad (Zelaya 2009).
Orden Heterobranchia

Familia Siphonariidae

Género Siphonaria

Siphonaria lessonii Blainville 1827

(Fig. 4 c-d).

Sinonimia: ver Dell (1971).

Lugar de colecta de los ejemplares: Estrecho de Magallanes (Fig. 6)

Distribución: En la costa chilena desde los $18^{\circ} \mathrm{S}$ hasta los $56^{\circ} \mathrm{S}$ (Zelaya 2009). En el Atlántico desde los $32^{\circ}$ hasta los $55^{\circ} \mathrm{S}$ (Morris \& Rosenberg 2005).

Comentario: Este gasterópodo pulmonado se encuentra en la zona intermareal. Tablado \& Lopez-Gappa (2001) encontraron que esta especie presenta una gran variabilidad morfométrica de la concha, atribuida principalmente por las características físicas y ambientales del hábitat. En relación a su biología, los adultos colocan masas ovígeras que contienen varias ovicápsulas, cada una de estas ovicápsulas contienen un único embrión que se desarrolla en su interior y eclosiona como una larva véliger (Zelaya 2009).

Clase Bivalvia

Orden Pteriomorphia

Familia Mytilidae

Género Mytilus

Mytilus edulis platensis d' Orbigny 1842

(Fig. 4 e).

Sinonimia: ver Reid \& Osorio (2000)

Lugar de colecta de los ejemplares: Estrecho de Magallanes (Fig. 6)

Distribución: Desde Iquique (Soot-Ryen 1959) hasta Bahía Orange (Rochebrune \& Mabille 1889). En el océano Atlántico en Islas Malvinas/Falkland (Dell 1964) e Isla de los Estados (USNM 2010).

Comentario: Puede confundirse con el bivalvo Choromytilus chorus (Molina 1782). Al respecto Zelaya (2009) comentaron que una forma de diferenciarlas es que $C$. chorus presenta un mayor tamaño, tiene las valvas comprimidas lateralmente y presenta tubérculos mas grandes en la charnela.

\section{Género Choromytilus}

Choromytilus chorus (Molina 1782)

(Fig. 4 f).

Sinonimia: ver Morris \& Rosenberg (2005).

Lugar de colecta de los ejemplares: Estrecho de Magallanes (Fig. 6)

Distribución: Desde los $7^{\circ} \mathrm{S}$ hasta lod $56^{\circ} \mathrm{S}$. (Reid \& Osorio 2000).

Comentario: Este organismo presenta valvas que pueden llegar a los $300 \mathrm{~mm}$ de longitud. Se le encuentra principalmente en costas expuestas en la zona del sublitoral entre los 4 y $20 \mathrm{~m}$, aunque tambien se le puede encontrar en el eulitoral bajo, y en zonas estuarinas con salinidades de 20 psu (Reid \& Osorio 2000). 


\section{Género Aulacomya}

Aulacomya atra (Molina, 1782)

(Fig. 4 g).

Sinonimia: ver Reid \& Osorio (2000).

Lugar de colecta de los ejemplares: Puerto Edén, Punta

Arenas y Estrecho de Magallanes (Fig. 6)

Distribución: Desde el Callao Peru (Soot-Ryen 1955) hasta Bahía Orange (Rochebrune \& Mabille 1889). En el Atlántico desde el Golfo San José (Zaixo 2004) hasta la Isla de los Estados (USNM 2010).

Comentario: Este bivalvo alcanza un largo de $200 \mathrm{~mm}$. Comúnmente se les puede encontrar desde los 40-79 m de profundidad, aunque también se le puede encontrar en el eulitoral bajo (Reid \& Osorio 2000). Estos mismos autores comentan que $A$. atra junto con otras especies de bivalvos como el Mytilus edulis platensis y el Choromytilus chorus son extraidos comercialmente por los pescadores.

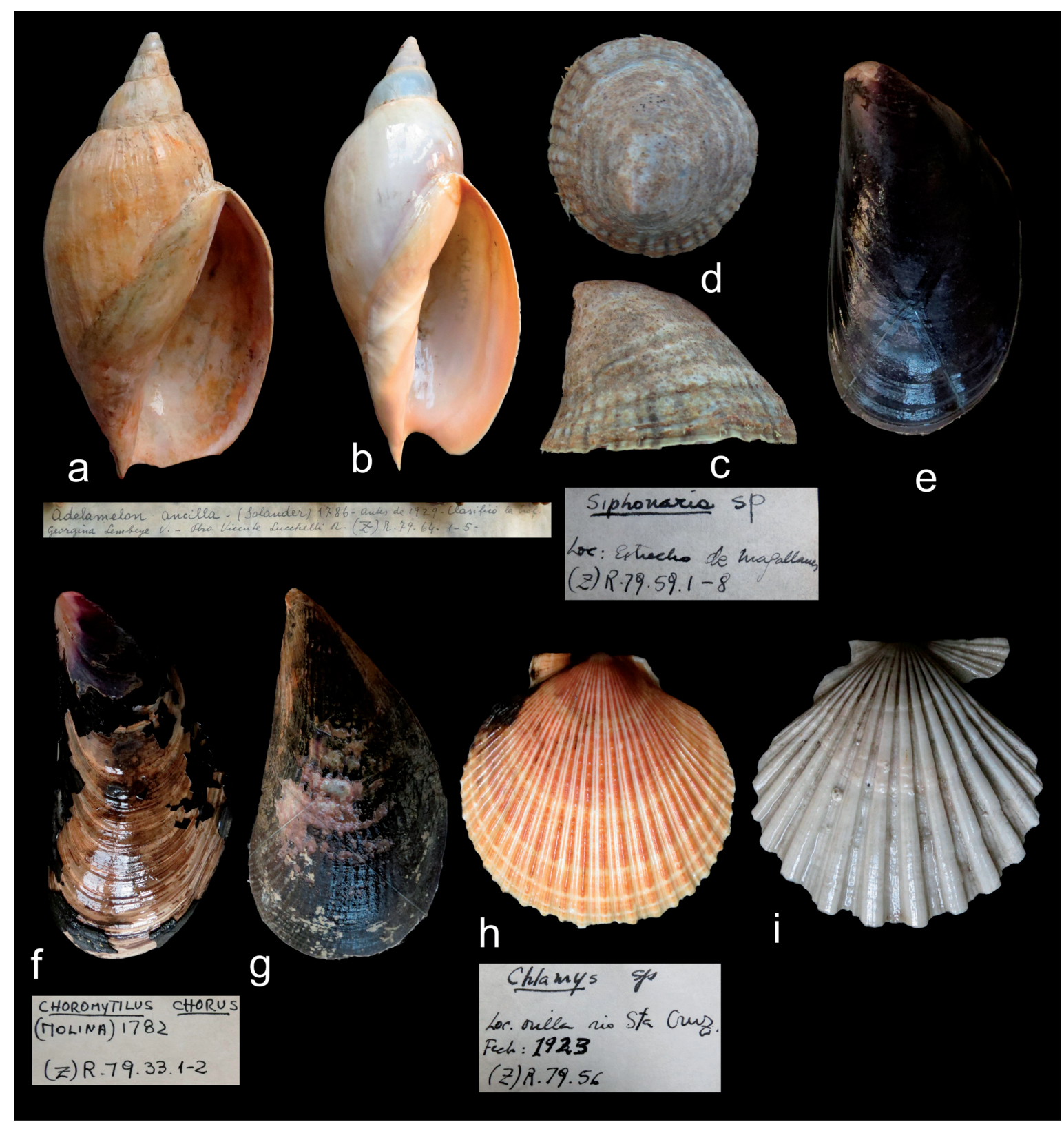

Figura 4. a) Odontocymbiola magellanica (228 mm; $95 \mathrm{~mm})$, b) Adelomelon ancilla (185 mm; $60 \mathrm{~mm})$, c-d) Siphonaria lesonii (25 mm; $22 \mathrm{~mm} ; 18 \mathrm{~mm})$, e) Mytilus edulis platensis $(37 \mathrm{~mm} ; 49 \mathrm{~mm}), \mathrm{f})$ Choromytilus chorus (150 mm; $74 \mathrm{~mm})$, g) Aulacomya atra (95 mm; 45 $\mathrm{mm})$, h) Zygochlamys patagonica $(86 \mathrm{~mm} ; 87 \mathrm{~mm})$, i) Aequipecten tehuelchus $(60 \mathrm{~mm} ; 60 \mathrm{~mm})$.

Figure 4. a) Odontocymbiola magellanica (228 mm; $95 \mathrm{~mm})$, b) Adelomelon ancilla (185 mm; $60 \mathrm{~mm})$, c-d) Siphonaria lesonii (25 mm; $22 \mathrm{~mm} ; 18 \mathrm{~mm})$, e) Mytilus edulis platensis $(37 \mathrm{~mm} ; 49 \mathrm{~mm}), \mathrm{f})$ Choromytilus chorus (150 mm; $74 \mathrm{~mm})$, g) Aulacomya atra (95 mm; 45 $\mathrm{mm}), \mathrm{g})$ Zygochlamys patagonica $(86 \mathrm{~mm} ; 87 \mathrm{~mm})$, i) Aequipecten tehuelchus $(60 \mathrm{~mm} ; 60 \mathrm{~mm})$. 
Familia Pectinidae

Género Zygochlamys

Zygochlamys patagonica (King \& Broderip 1832)

(Fig. 4 h).

Sinonimia: ver Cárdenas et al. (2008).

Lugar de colecta de los ejemplares: Río Gallegos e Isla Dawson (Fig. 6)

Distribución: Desde Chiloé hasta Cabo de Hornos (Waloszek 1984). En el océano Atlántico desde los $34^{\circ} \mathrm{S}$ hasta los $55^{\circ} \mathrm{S}$ (Morris \& Rosenberg 2005).

Comentario: En relación a su hábitat, Zelaya (2009), comento que esta especie es epifaunal, presentes en fondos arenosos, fangosos, de grava y conchilla. Los ejemplares adultos habitan apoyados sobre la valva derecha; y los juveniles aparecen como epibiontes de algas. Recientemente se registraron juveniles habitando en frondas del alga roja Gigartina skottsbergii (Rosenfeld et al. 2015).

\section{Género Aequipecten}

Aequipecten tehuelchus (d'Orbigny, 1842)

(Fig. 4 i).

Sinonimia: ver Waloszek (1984).

Lugar de colecta de los ejemplares: Rada Tily (Fig. 6)

Distribución: Desde los $50^{\circ} \mathrm{S}$ hasta los $55^{\circ} \mathrm{S}$ (Valdovinos 1999). En el océano Atlántico desde los $21^{\circ} \mathrm{S}$ hasta los $53^{\circ} \mathrm{S}$ (Morris \& Rosenberg 2005).

Comentario: Respecto a su ecología, Zaixo (2004) comentó que los bancos de A. tehuelchus, se presentan principalmente sobre fondos blandos, con preferencia en aquellos que contienen gránulos que sirven para la fijación bisal de los individuos. Esta especie se halla dispersa sobre el fondo (Zaixo 2004).

Orden Heterodonta

Familia Mactridae

\section{Género Mulina}

Mulinia edulis (King \& Broderip 1832)

(Fig. 5 a-b).

Sinonimia: ver Signorelli \& Pastoriono (2011)

Lugar de colecta de los ejemplares: Estrecho de Magallanes (Fig. 6)

Distribución: Desde Ecuador hasta el Canal Beagle (Signorelli \& Pastoriono 2011). En el océano Atlántico desde los $52^{\circ} \mathrm{S}$ hasta $\operatorname{los} 55^{\circ} \mathrm{S}$ (Morris \& Rosenberg 2005) Comentario: Signorelli \& Pastoriono (2011), comentaron que una característica para diferenciar esta especie con la especie Mulinia byronensis Gray 1837, es que esta última presenta una concha más elongada y que el seno palial ocupa 1/3 del largo de la concha, mientras que en $M$. edulis el seno palial ocupa la mitad del largo de la concha.

Familia Pharidae

Género Ensis
Ensis macha (Molina 1782)

(Fig. 5 c).

Sinonimia: ver Reid \& Osorio (2000)

Lugar de colecta de los ejemplares: Rada Tily (Fig. 6)

Distribución: Desde los $27^{\circ} \mathrm{S}$ hasta los $54^{\circ} \mathrm{S}$ (Reid \& Osorio 2000). Desde los $52^{\circ} \mathrm{S}$ hasta los $55^{\circ} \mathrm{S}$ (Morris \& Rosenberg 2005).

Comentario: En relación a su morfología, Reid \& Osorio (2000) señalaron que presenta un color blanco amarillento fuerte; sus valvas pueden llegar a medir $211 \mathrm{~mm}$ de largo. Generalmente se le puede encontrar entre 2-10 $\mathrm{m}$ de profundidad, en fondos de arena, limo y arenas gruesas; aunque se han encontrado juveniles a los $26 \mathrm{~m}$ de profundidad en Bahía Valparaiso (Reid \& Osorio 2000).

Familia Veneridae

Género Ameghinomya

Ameghinomya antiqua (King \& Broderip 1832)

(Fig. 5 d).

Sinonimia: ver Reid \& Osorio (2000).

Lugar de colecta de los ejemplares: Bahía Santa María

(Fig. 6)

Distribución: Desde los $18^{\circ} \mathrm{S}$ hasta los $56^{\circ} \mathrm{S}$ (Zelaya 2009).

En el océano Atlántico desde los $34^{\circ} \mathrm{S}$ Hasta los $54^{\circ} \mathrm{S}$ (Morris \& Rosenberg 2005).

Comentario: Es una especie de hábito infaunal, generalmente en pozas de marea, fondos arenosos, areno-fangoso o de guijarros, y en bancos de mejillones, habitando comúnmente entre el intermareal y los $65 \mathrm{~m}$ de profundidad (Zelaya 2009). Al igual que la almeja Retrotapes exalbidus, son extraidas comercialmente por los pescadores (Reid \& Osorio 2000).

\section{Género Tawera}

Tawera elliptica (Hupé 1854)

(Fig. 5 e-f).

Sinonimia: ver Gordillo (2006).

Lugar de colecta de los ejemplares: Estrecho de Magallanes (Fig. 6)

Distribución: Desde Valparaíso (Osorio \& Bahamonde 1970) hasta el Cabo de Hornos (USNM 2010). En el océano Atlántico en Islas Malvinas/Falkland (Linse 1997) e Isla de los Estados (USNM 2010).

Comentario: Se caracteriza por presentar un margen interno crenulado con tres fuertes dientes cardinales en cada válvula y una coloración violácea interna (Reid \& Osorio 2000). En recientes estudios se ha registrado habitando en praderas de Gigartina skottsbergii (Rosenfeld et al. 2015).

\section{Género Retrotapes}

Retrotapes exalbidus (Dillwyn 1817)

(Fig. 5 g).

Sinonimia: ver Reid \& Osorio (2000).

Lugar de colecta de los ejemplares: Islas Malvinas/Falkland (Fig. 6) 
Distribución: Desde los $18^{\circ} \mathrm{S}$ hasta los $56^{\circ} \mathrm{S}$ (Zelaya 2009). En el océano Atlántico desde los $34^{\circ} \mathrm{S}$ hasta los $55^{\circ} \mathrm{S}$ (Morris \& Rosenberg 2005).

Comentario: Presenta variabilidad en la concha y en su escultura (Reid \& Osorio 2000), pudiéndosele confundir con ejemplares de la especie Tawera elliptica. Al respecto, Zelaya (2009), mencionaron que una forma de diferenciarlos es que el bivalvo $T$. elliptica presenta una coloración interior violácea. Esta especie es de hábito infaunal, en fondos arenosos, fangosos $\mathrm{y}$ areno-fangosos, presentando un rango batímetrico que va desde los $5 \mathrm{~m}$ a los $150 \mathrm{~m}$ de profundidad (Zelaya 2009).

Familia Gaimardiidae

Género Gaimardia

Gaimardia trapesina (Lamarck 1819)
(Fig. 5 h).

Sinonimia: ver Morris \& Rosenberg (2005).

Lugar de colecta de los ejemplares: Estrecho de Magallanes (Fig. 6)

Distribución: Desde los $37^{\circ} \mathrm{S}$ (Zelaya 2009) hasta Bahía Orange (Rochebrune \& Mabille 1889). En el océano Atlántico desde los $28^{\circ} \mathrm{S}$ (Morris \& Rosenberg 2005) hasta la Isla de los Estados (USNM 2010).

Comentario: Se caracteriza porque generalmente se la encuentra como epibionte sobre frondas superficiales de algas pardas, particularmente Macrocystis pyrifera (Ralph \& Maxwell 1977). En recientes estudios también se le ha registrado habitando sobre frondas de Gigartina skottsbergii (Rosenfeld et al. 2015).

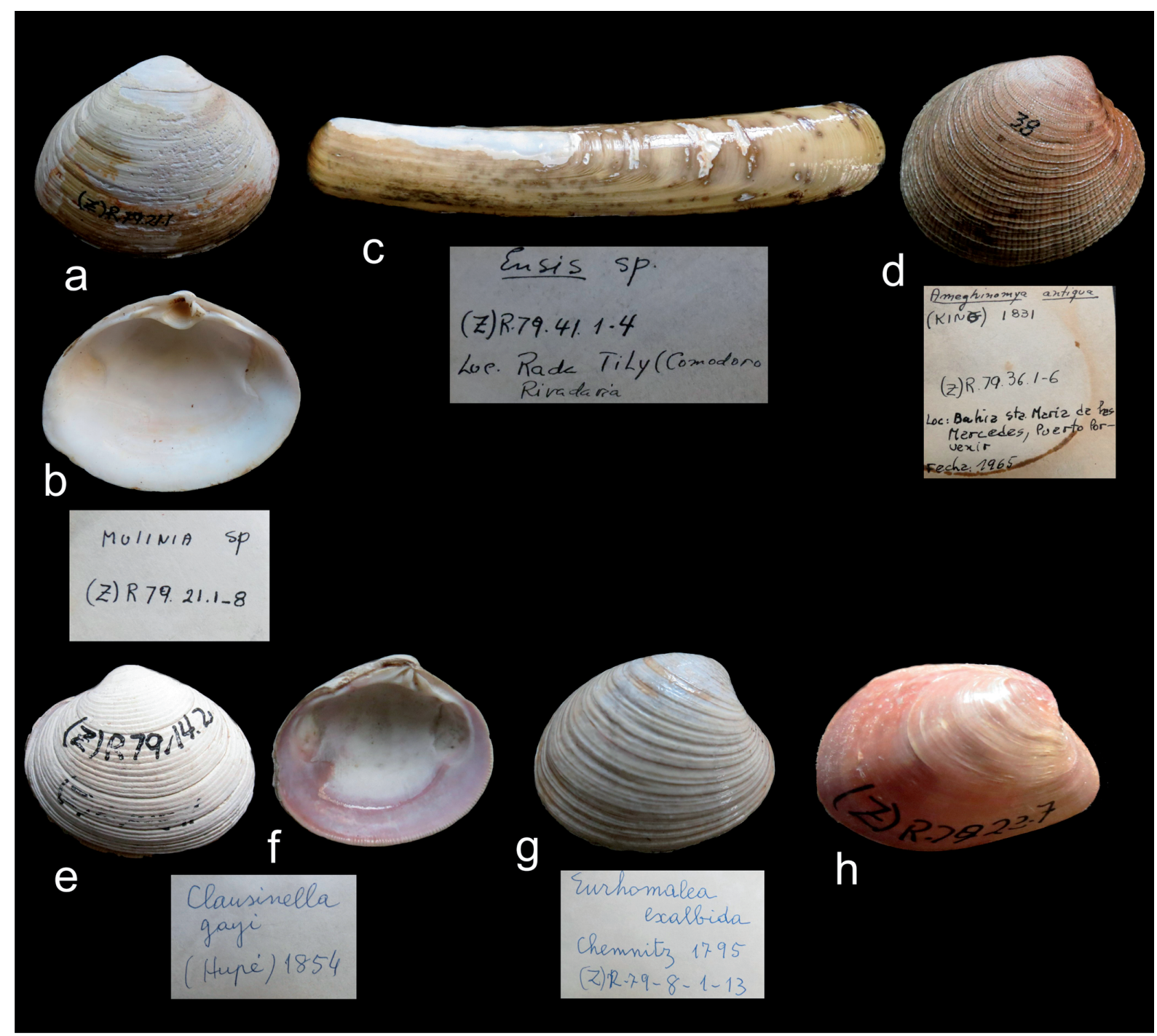

Figura 5. a-b) Mulinia edulis (37 mm; $49 \mathrm{~mm})$, c) Ensis macha (160 mm; $28 \mathrm{~mm})$, d) Ameghinomya antiqua (35 mm; $40 \mathrm{~mm})$, e-f) Tawera elliptica $(30 \mathrm{~mm} ; 35 \mathrm{~mm}), \mathrm{g})$ Retrotapes exalbidus $(55 \mathrm{~mm} ; 60 \mathrm{~mm})$, h) Gaimardia trapesina $(15 \mathrm{~mm} ; 20 \mathrm{~mm})$.

Figure 5. a-b) Mulinia edulis (37 mm; $49 \mathrm{~mm})$, c) Ensis macha (160 mm; $28 \mathrm{~mm})$, d) Ameghinomya antiqua (35 mm; $40 \mathrm{~mm})$, e-f) Tawera elliptica (30 mm; $35 \mathrm{~mm})$, g) Retrotapes exalbidus (55 mm; $60 \mathrm{~mm})$, h) Gaimardia trapesina (15 mm; $20 \mathrm{~mm})$. 


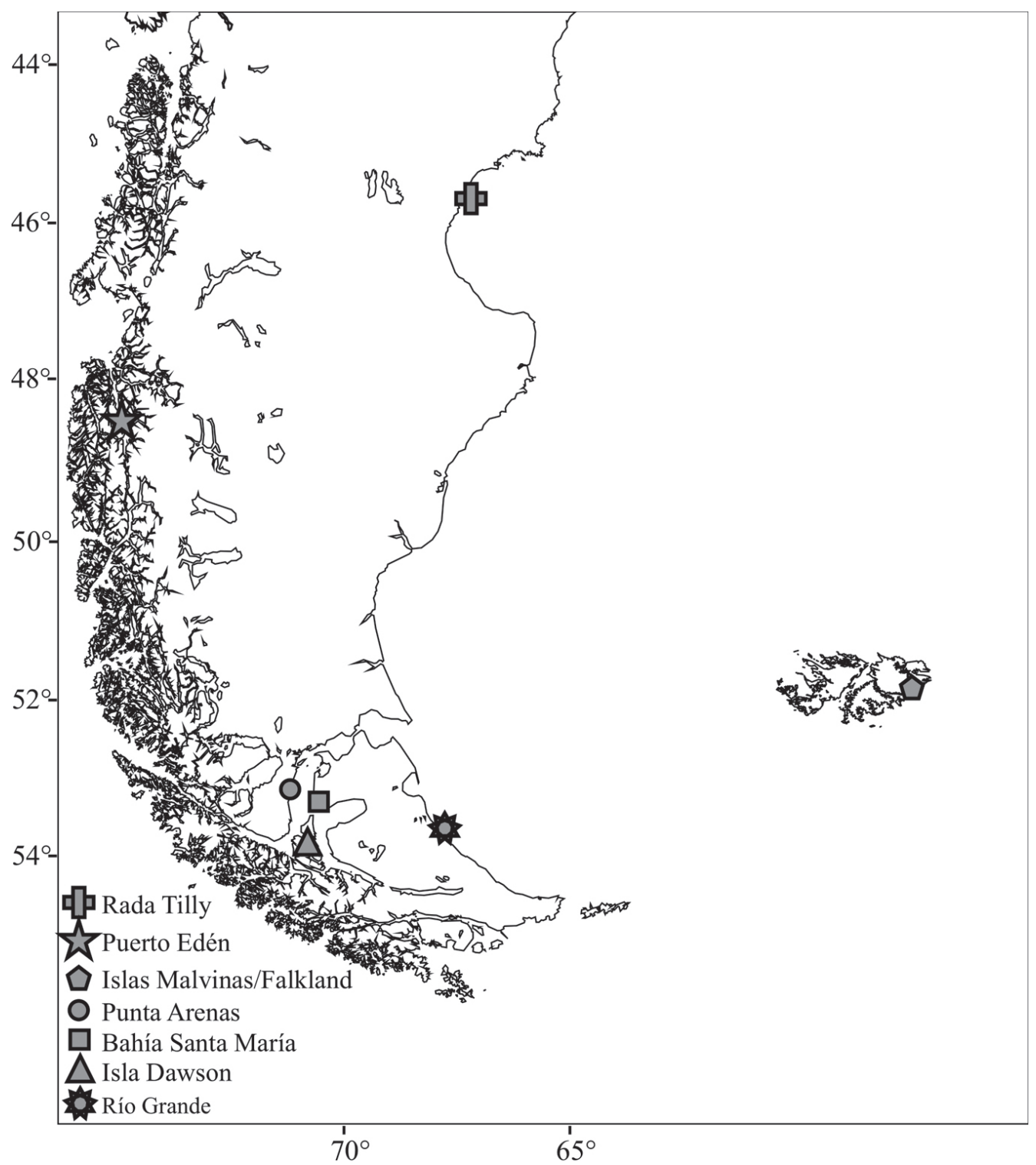

Figura 6. Lugar de colecta de los ejemplares de moluscos depositados en el Museo Maggiorino Borgatello (Punta Arenas, Chile).

Figure 6. Place of collecting specimens of mollusks deposited in the Museo Maggiorino Borgatello (Punta Arenas, Chile).

\section{DISCUSIÓN}

Para la región de Magallanes la presencia de esta colección de moluscos de altas latitudes en el Museo Maggiorino Borgatello marca un hito, tanto para la ciencia y la historia de esta región, ya que esta es una forma de conservación del patrimonio natural y cultural de la zona. Tal ejemplo se puede apreciar en los trabajos de Knebelsberger et al. (2005) y Dijkstra \& Köhler (2008). La Región de Magallanes se encuentra a la espera de nuevas investigaciones multidiciplinarias, que se enfoquen en dar a conocer el valor propio, ecológico y cultural de los moluscos que habitan estas altas latitudes.
REGISTROS Y DISTRIBUCIÓN DE LAS ESPECIES

Todos los ejemplares depositados en este museo corresponden a especies previamente registradas en la provincia Biogeográfica Magallánica (Valdovinos 1999, Linse 1999), por lo tanto, no hay ninguna especie presente en esta colección que este fuera de sus rangos de distribución. No obstante, cabe destacar los registros de la especies Buccinanops paytensis y Choromytilus chorus para el Estrecho de Magallanes, ya que, ninguna de estas especies ha vuelto a ser reportada en trabajos contemporáneos en el Estrecho de Magallanes. (Mutschke et al. 1998, Linse 2002, Rios et al. 2003, Rios et al. 2007, Aldea \& Rosenfeld 2011, Aldea et al. 2011, Rosenfeld et al. 2015). Por lo que, si 
efectivamente hace 100 años era común la colecta de estas especies en el Estrecho de Magallanes algo podría haber generado la disminución de sus poblaciones. Otra posible alternativa es que a estas latitudes estas especies son de baja ocurrencia. Finalmente para ir pudiendo dilucidar estas interrogantes sería importante evaluar en la actualidad su presencia en esta zona.

\section{RiQueZA DE ESPECIES}

De las 959 especies marinas (671 gasterópodos, 226 bivalvos y 63 poliplacóforos) citadas en la base taxonómica de Valdovinos (1999) para el territorio nacional de Chile, incluyendo especies antárticas y de islas oceánicas, un total de 437 especies (302 gasterópodos, 99 bivalvos y 36 poliplacóforos) las registró dicho autor para la franja latitudinal $50-55^{\circ} \mathrm{S}$. Al respecto, las 37 especies presentes en el museo (26 gasterópodos y 11 bivalvos) corresponden al $\sim 4 \%$ de las especies citadas para Chile ( $\sim 4 \%$ de los gasterópodos totales y $\sim 5 \%$ de bivalvos totales). Regionalmente, corresponden a un $\sim 8 \%$ de las especies citadas por Valdovinos (1999) para la franja latitudinal $50-55^{\circ} \mathrm{S}(\sim 9 \%$ de los gastrópodos y $\sim 11 \%$ de bivalvos).

Utilizando la base de datos propuesta por Linse (1999) para la región Magallánica, definida como la plataforma patagónica al sur de $41^{\circ} \mathrm{S}$ en los márgenes Pacífico y Atlántico de Sudamérica, fueron citadas 381 especies marinas (250 gasterópodos y 131 bivalvos, no estando incluida la clase Polyplacophora debido a dificultades taxonómicas del grupo). Según dicha base de datos, las 37 especies de gasterópodos y bivalvos presentes en el museo corresponden al $\sim 10 \%$ ( $\sim 10 \%$ de los gasterópodos totales y $\sim 8 \%$ de bivalvos totales) de las especies citadas para estas clases.

En esta colección de moluscos se destaca el gran número de especies de interés comercial presentes en el museo, ya que de las 37 especies presentes en esta colección, 19 corresponden a especies de interés comercial ( $\sim 51 \%$ de la colección). Tomando la información publicada por Osorio (2002), de moluscos de interés comercial, ella reportó un total de 132 especies para la costa chilena. Las 19 especies presentes en el museo corresponden al $\sim 14 \%$ de las especies de interés comercial citadas para Chile. En especial se destaca el gran número de ejemplares de Concholepas concholepas, Adelomelon ancilla, Trophon geversianus, Nacella magellanica, Tawera elliptica, Retrotapes exalbidus Zygochlamys patagonicus y Aulacomya atra, contabilizándose más de 100 ejemplares depositados en el museo y que corresponden al $31 \%$ de la colección total.

\section{AGRADECIMIENTOS}

Los autores expresan su gratitud al Museo Maggiorino Borgatello y a todo su personal, por facilitar la colección malacológica, brindando óptimas condiciones para el desarrollo del estudio. Al Laboratorio de Macroalgas Antárticas y Subantárticas de la Universidad de Magallanes por brindar soporte a la investigación. Se reconoce, además, el aporte de la Fundación CEQUA, en equipos y gestión para el cumplimiento de los objetivos del estudio. S. Rosenfeld y J. Marambio agradecen al Programa de Magíster en Ciencias con Mención en Manejo y Conservación de Recursos Subantárticos de la Universidad de Magallanes y a las becas de Magíster del Proyecto ICM, código P05-002 y código PFB -23-2008 otorgada por el Instituto de Ecología y Biodiversidad.

\section{BIBLIOGRAFÍA}

Aldea, C. \& Valdovinos, C. 2005. Moluscos del intermareal rocoso del centro-sur de Chile $\left(36^{\circ}-38^{\circ} \mathrm{S}\right)$ : taxonomía y clave de identificación. Gayana 69(2):364-396.

Aldea, C. \& Troncoso, J. S. 2010. Remarks on the genus Trophon (s.l.) Montfort, 1810 (mollusca: gastropoda: muricidae) in the southern ocean and adjacent areas. Thalassas 26(2):4773.

AldeA, C. \& Rosenfeld, S. 2011. Moluscos intermareales de la Playa Buque Quemado (Estrecho de Magallanes, Chile). Revista de Biología Marina y Oceanografía 46(2):115-124.

Aldea, C., Rosenfeld S. \& Cárdenas, J. 2011. Caracterización de la diversidad de moluscos bentónicos sublitorales en la isla Carlos III y áreas adyacentes, Estrecho de Magallanes, Chile. Anales del instituto de la Patagonia 39(2):73-89.

Allmon, W.D. 1990. A review of the Bullia group (Gastropoda: Nassariidae) with comments on its evolution, biogeography and phylogeny. Bulletin of American Paleontology 99(335):1-179.

Aranzamendi, M.C., Gardenal, C.N., Martin, J.P. \& Bastida, R. 2009. Limpets of the genus Nacella (Patellogastropoda) from the Southwestern Atlantic: species identification based on molecular data. Journal of Molluscan Studies 75:241-251.

Avaca, S., Narvarte, M. \& Martin, P. 2010. Description of the radula of Buccinanops globulosus (Neogastropoda, Nassariidae) and an assessment of its variability in northern patagonian Gulfs. Malacologia 53(1):175-183.

Cárdenas, J., Aldea C. \& Valdovinos C. 2008. Chilean marine mollusca of Northern Patagonia collected during the CIMAR-10 Fjords Cruise. Gayana 72(2):202-240.

Castellanos, Z.A. 1956. Catálogo de los poliplacóforos argentinos y de aguas vecinas al Estrecho de Magallanes. Revista del Museo de La Plata 6:465-486.

Castellanos, Z.A. \& Fernández, D. 1976. Los géneros Calliostoma y Neocalliostoma del mar argentino con especial referencia al área subantártica. Revista del Museo de la Plata 12(116):135-156. 
Castellanos, Z.A. \& Landoni, N.A. 1988. Catálogo descriptivo de la malacofauna marina Magallánica 2. Archigastropoda. Comisión de Investigaciones Científicas Buenos Aires. 40pp

Dell, R.K. 1964. Antarctic and sub-Antarctic Mollusca: Amphineura, Scaphopoda and Bivalvia. Discovery Reports 33:99-250.

Dell, R.K. 1971. The marine Mollusca of the Royal Society Expedition to southern Chile, 1958- 1959. Records of the Dominion Museum 7(17):155-233.

Dijkstra, H. 1999. Type specimens of Pectinidae (Mollusca: Bivalvia) described by Linnaeus (1758-1771). Zoological Journal of the Linnean Society 125:383-443.

Dijkstra, H. \& KöHLer, F. 2008. An annotated catalogue of Recent Pectinoidea (Mollusca, Pectinidae and Propeamussiidae) Type material in the Museum of Natural History, Humboldt University, Berlin. Zoosystematics and Evolution 84(1):31-44.

Gallardo, C. 1979. Especies gemelas del género Crepidula (Gastropoda, Calyptraeidae) en la costa de Chile; una redescripción de $C$. dilatata Lamarck y descripción de C. fecunda n. sp. Studies on the Neotropical Fauna and Environment 14:215-226.

González-Wevar, C., Nakano, T., Cañete J. I. \& Poulin, E. 2010. Molecular phylogeny and historical biogeography of Nacella (Patellogastropoda: Nacellidae) in the Southern Ocean. Molecular Phylogenetics and Evolution 56:115124.

González-Wevar, C., Nakano, T., Cañete, J. I. \& Poulin, E. 2011. Concerted genetic, morphological and ecological diversification in Nacella limpets in the Magellanic Province. Molecular Ecology 20:1936-1951.

Gordillo, S. 2006. The presence of Tawera gayi (Hupé in Gay, 1854) (Veneridae, Bivalvia) in southern South America: Did Tawera achieve a Late Cenozoic circumpolar traverse? . Palaeogeography, Palaeoclimatology, Palaeoecology 240:587-601

Knebelsberger, T., Schwabe, E. \& Schrödl, M. 2005. Molluscan types of the Bavarian state collection of Zoology Munich (ZSM) Part. 1. Polyplacophora, Scaphopoda, Cephalopoda. Spixiana 28(2):97-108.

KING, P.P. \& BroderIP, W.J. 1832. Description of the Cirripedia, Conchifera and Mollusca, in a collection formed by the officers of H.M.S. Adventure and Beagle employed between the years 1826 and 1830 in surveying the southern coasts of South America. Zoological Journal 5:332-349.

Melvill, J.C. \& Standen, P. 1907. The Marine Mollusca of the Scottish National Antarctic Expedition. Transactions of the Royal Society of Edinburgh 46:119-157.

Melvill, J.C. \& Standen, P. 1914. Notes on Mollusca collected in the Northwest Falklands by Mr. Rupert Vallentin, F.L.S. with descriptions of some new species. Annals and Magazine of Natural History (8)13:110-36.

McLean, J.H. 1984. Systematics of Fissurella in the Peruvian and Magellanic faunal provinces (Gastropoda: Prosobranchia). Contributions in Science, Natural History Museum of Los Angeles County 354:1-70.

Morris, P.J. \& Rosenberg, G. 2005. Search interface and documentation for Malacolog, an online database of Western Atlantic marine Mollusks. URL: http:// www. malacolog.org. Accesado Octubre 15, 2014.

Mutschke, E., Ríos C. \& Montiel, A. 1998. Situación actual de la macrofauna presente en el intermareal de bloques y cantos de Bahía Laredo, Estrecho de Magallanes. Anales del Instituto de la Patagonia, Serie Ciencias Naturales 26:5-29.

LAmY, E. 1905. Gastropodes prosobranches recueillis par l'Expédition Antarctique Française du Dr. Charcot. Bulletin du Museum d'Histoire Naturelle 11:475-483.

LinSE, K. 1999. Mollusca of the Magellan region. A cheklist of the species and their distribution. Scientia Marina 63(1):399407.

LinSE, K. 2002. The shelled magellanic Mollusca: with special reference to biogeographic relations in the Southern Ocean. Theses Zoologicae 34:1-252.

Osorio, C., Atria, J. \& Mann, S. 1979. Moluscos marinos de importancia económica en Chile. Biología Pesquera, Chile 11:3-47.

Osorio, C. 2002. Moluscos marinos en Chile: especies de importancia económica. Facultad de Ciencias, Universidad de Chile, Santiago. 211 pp.

Pastorino, G. 2005A. Recent Naticidae (Mollusca: Gastropoda) from the Patagonian Coast. The Veliger 47(4):225-258.

Pastorino, G. 2005B. A revision of the genus Trophon Monfort, 1810 (Gastropoda: Muricidae) from southern South America. The Nautilus 119(2):55-82.

Pastorino, G. \& Harasewych, M.G. 2000. A revision of the Patagonian genus Xymenopsis Powell 1951. The Nautilus 114(2):38-58.

Pastorino, G. \& Penchaszadeh, P.E. 2002. Spawn of the Patagonian gastropod Pareuthria plumbea (Philippi, 1844) (Buccinidae). The Nautilus 116(3):105-108.

Powell, A.W.B. 1951. Antarctic and Subantarctic Mollusca: Pelecypoda and Gastropoda. Discovery Reports 26:47196.

Ralph, R. \& MaXwell, H. 1977. The oxigen consumption of the Antarctic Lamellibranch Gaimardia trapesina trapesina in relation to cold adaptation in polar invertebrates. British Antarctic Survey Bulletin 45:4-46.

Reid, D.G. \& Osorio, C. 2000. The shallow-water marine mollusca of the Estero Elefantes and Laguna San Rafael, southern Chile. Bulletin of the Natural History Museum of London, Zoology 66(2):109-146.

Ríos, C., Mutschke, E. \& Morrison, E. 2003. Biodiversidad bentónica sublitoral en el estrecho de Magallanes, Chile. Revista de Biología Marina y Oceanografía 38(1):1-12.

Ríos, C., Arntz, W.E., Gerdes, D., Mutschke, E. \& Montiel, A. 2007. Spatial and temporal variability of the benthic assemblages associated to the holdfasts of the kelp Macrocystis pyrifera in the Straits of Magellan, Chile. Polar Biology 31:89-100.

Rochebrune, A.T. \& Mabille, J. 1889. Mollusques. Mision Scientifique du Cap Horn. VI, Zoologie, Paris. 129 pp.

Rosenfeld, S., Aldea C. \& OJeda, J. 2011. Nuevos antecedentes sobre la biología y distribución del gasterópodo Margarella expansa (Sowerby, 1838). Amici Molluscarum 19:19-26.

Rosenfeld, S. 2012. Composición y abundancia estacional de ensambles de moluscos presentes en dos praderas naturales de Gigartina skottsbergii (Rhodophyta, Gigartinales) en el Estrecho de Magallanes, Chile. Tesis de Pregrado, Departamento de Ciencias y Recursos Naturales, 
Universidad de Magallanes, Punta Arenas, Chile, 118pp.

Schwabe, E. 2010. Illustrated summary of chiton terminology. Spixiana, 33(2): 171-194.

Signorelli, J. \& Pastorino, G. 2011. Revision of the Magellanic Mactridae Lamarck, 1809 (Bivalvia: Heterodonta). Zootaxa 2757:47-67.

Soot-Ryen, T. 1955. A Report on the Family Mytilidae. Allan Hancock Pac. Exped. 20:1-154.

Soot-Ryen, T. 1959. Pelecypoda. Reports of the Lund University Chile Expedition 1948-49. Lunds Universitets Lrsskrift Lund 55(6):1-86.

Smith, E.A. 1905. On a small collection of Mollusca from Tierra del Fuego. Proceeding of The Malacological Society of London VI:333-339.

Strebel, H. 1905A. Beiträge zur Kenntnis der Molluskenfauna der Magalhaen-Provinz, II. Die Trochiden. Zoologische Jahrbücher Suppl. 8:121-166.

Strebel, H. 1905B. Beiträge zur Kenntnis der Molluskenfauna der Magalhaen-Provinz. No 3. Zoologische Jahrbücher. Abteilung für Systematik, Geographie und Biologie der Tiere 22:575-666.

Strebel, H. 1906. Beiträge zur Kenntnis der Molluskenfauna der Magalhaen-Provinz. No 4. Zoologische Jahrbücher. Abteilung für Systematik, Geographie und Biologie der Tiere 24:91-174.

Strebel, H. 1908. Die Gastropoden (mit Ausnahme de nackten Opisthobranchier). Wissenschaftliche Ergebnisse der Schwedischen Südpolar-Expedition 1901-1903 6(1):1111,6 pls.

SuArez, A.V. \& Tsutsui, N.D. 2004. The value of museum collections for research and society. BioScience 54:66-74.

Tablado, A. \& López-Gappa, J. 2001. Morphometric diversity of the pulmonate limpet Siphonaria lessoni in different coastal environments. Scientia Marina 65(1):33-41.

USNM. 2010. National Collection of the Smithsonian National
Museum of Natural History, USNM. URL: http:// invertebrates.si.edu/. Accesado: Agosto 20, 2014

Urra, A., Oliva, D. \& Sepúlveda, M. 2007. Use of a Morphometric Analysis to Differentiate Adelomelon ancilla and Odontocymbiola magellanica (Caenogastropoda: Volutidae) of Southern Chile. Zoological Studies 46(3):253-261.

Valdovinos, C. \& Rüth, M. 2005. Nacellidae limpets of the southern end of South America: taxonomy and distribution. Revista Chilena de Historia Natural 78:497-517.

Valdovinos, C. 1999. Biodiversidad de moluscos chilenos: Base de datos taxonómica y distribución. Gayana 63(2):111164.

Walozeck, D. 1984. Variabilität, Tazonomie und Verbreitung von Vhlamys patagonica (King and Broderip, 1832) und Anmerkungen zu weiteren Chlamys-Arten von der Südspitze Süd-Amerikas (Mollusca, Bivalvia, Pectinidae). Verhandlungen des Naturwissenschaftlichen Vereins inHamburg 27:207-276.

WiLson, E.O. 2003. The encyclopedia of life. Trends in Ecology and Evolution 18:77-80.

ZAIXo, E. 2004. Bancos de cholga Aulacomya atra atra (Molina) (Bivalvia: Mytilidae) del golfo San José (Chubut, Argentina): Diversidad y relaciones con facies afines. Revista de Biología Marina y Oceanografía 39(2):61-78.

ZelayA, D. 2004. The genus Margarella Thiele, 1893 (Gastropoda: Trochidae) in the southwestern Atlantic ocean. The Nautilus 118(3):112-120.

ZelayA, D.G. 2005. Systematics and biogeography of marine gastropods molluses from South Georgia. Spixiana 28(2):109-139.

Zelaya, D.G. 2009. Gastropoda - Gasterópodos. En: Fauna Marina Bentónica de la Patagonia Chilena (Eds. Häussermann, V. \& G. Försterra ). pp. 461-504. Nature in Focus, Chile.

Recibido: 04.08.15

Aceptado: 20.04.16 